\title{
On Inverted Cones and Their Elastic Creases
}

\author{
Keith A. Seffen
}

November 16, 2016

kas14@cam.ac.uk: Advanced Structures Group Laboratory, Department of Engineering, University of Cambridge, UK

\begin{abstract}
We study the elastic inversion of a right circular cone, in particular, the uniform shape of the narrow crease which divides its upright and inverted parts. Our methodology considers a cylindrical shell analogy for simplicity where the crease is the boundary layer deformation. Solution of its governing equation of deformation requires careful crafting of the underlying assumptions and boundary conditions in order to reveal an expression for the crease shape in closed form. We can then define the characteristic width of crease exactly, which is compared to a geometrically nonlinear, large displacement finite element analysis. This width is shown to be accurately predicted for shallow and steep cones, which imparts confidence to our original assumptions. Using the shape of crease, we compute the strain energy stored in the inverted cone, in order to derive an expression for the applied force of inversion by a simple energy method. Again, our predictions match finite element data very well. This study may complement other studies of creases traditionally formed in a less controlled manner, for example, during crumpling of lightweight sheets.
\end{abstract}

Keywords: cone, inversion, elastic, crease, size, inverting force

\section{Introduction}

Indenting a thin, doubly curved elastic surface can result in local inversion, where a dimple forms and spreads over the surface. For the case of a point-loaded spherical cap, the dimple is axisymmetrical and bounded by a planar circular ridge. In the limit of zero cap thickness, the ridge acquires infinitesimal width as the bending stiffness diminishes; furthermore, the dimple is a perfect reflection of the initial region above the current ridge plane. This simplified but immediate view of the partially inverted shape was first proposed by Pogorelov [1]. As we increase the thickness, the ridge circle expands to become a smooth open torus, or crease, which seamlessly interconnects the upright and inverted parts. Tracing out a meridian on the surface gives a clear indication of the crease extent but where it precisely begins or ends is 
not immediately obvious. Most of the elastic deformation, however, is concentrated in the crease, so knowing its shape precisely is key to finding other features of the problem such as the applied forces in equilibrium. Often, the crease is endowed with a uniform shape because of its much smaller "size" - usually a constant radius of curvature [2], which allows the crease width to be simply written in terms of its meridional arc-length or its projected width onto the ridge plane. A straightforward dimensional or energy analysis is then possible with the familiar scaling law that width depends on the square root of the product of shell thickness and spherical radius. Determining the constant of proportionality in this expression typically requires data from experiments or numerical simulations, so the absolute width is often an approximate, if dimensionally correct, expression.

A more recent and thorough examination of the Pogorelov proposition appears in [3], which also furnishes a historical review of spherical cap inversion. They revise upwards the number of latitudinal "regimes" to seven, with five alone describing the inverted shape between the central loading point and the ridge, followed by the ridge itself and the original part beneath. These regimes are found from an elegant asymptotic analysis of the governing equation of axisymmetrical deformation, which is compared against its full numerical solution and finite element analysis. There is a richness of results, however, the crease extent is expressed only in order-ofmagnitude terms because it is not of explicit interest. Here, we examine the precise shape of a similar crease formed during inversion of a right circular cone. Figure 1 indicates some partially inverted shapes obtained from the finite element analysis of the next section. Three major geometrical regimes are evident: upright and inverted sections which are largely undeformed, and a narrow crease. We can surmise a Pogorelov-type profile in the limit of zero thickness, where the cutaway in Fig. 1(c) shows the more heavily strained area pertaining to the crease diverging from this profile superimposed. Axisymmetry is prescribed at all times during computation even though new studies suggest the possibility of secondary "buckling" of the circular ridge into a rough polygonal outline in practice [4]. We shall be concerned only with solving for the shape of an axisymmetrical crease using the linear governing equation of deformation for a cylindrical shell.

Such an approach would appear to flout the major effects of geometrical nonlinearity and initial cone shape upon the crease shape, but it greatly reduces the complexity of analysis and enables accurate closed-form solutions provided two key assumptions are upheld. First, a Pogorelov viewpoint for a partially inverted cone of zero thickness is applicable: this then provides a coordinate reference for actual displacements. Second, that the meridional span of major displacements around the ridge-line is very short such that the curvature of the underlying Pogorelov reference remains approximately constant i.e. cylindrical, over this span. The validity 
of this approach is then tested in two subtle ways. When the cone is steep, it is naturally close to a cylinder but across the ridge there can be large rotations - up to $180^{\circ}$, depending on the initial geometry; on the other hand, a shallow cone is less like a cylinder but has much smaller displacement gradients in keeping with the "shallow" shell requirement of the linear governing equation. Remarkably accurate results however emerge for a range of conical geometries. We then determine an expression for the inverting force applied to the apex, which is usually intractable in other "elastic" studies: during plastic inversion of metal cones and other axisymmetrical shells, where crease localisation can occur too, the driving force is usually amenable in closed form but the underlying formation and propagation of creases are very different to here, see $e . g .[5]$.

We shall not quantify the formation stage for this itself is challenging. For example, conical inversion begins with turning the sharp apex inside out, which is impractical and leads to computational difficulties because of the singular geometry. But we must marshal inversion in some way, so we "cheat" at the very start by smoothing the apex to form a very small, localised spherical cap [6]. Under a central point force, this region locally inverts early on in the deformation profile, enabling conical inversion to become well developed - the starting point of our study. We consider a range of cone angles, including a tube in the extreme, and we highlight representative behaviour, in particular, our definition of crease width according to how the ridge strains attenuate. We then present our analytical model and compare predictions with finite element data before concluding with a brief discussion.

\section{Finite element analysis}

The commercial software package used in Fig. 1 and throughout is ABAQUS [7]. Elements are axisymmetrical two-noded linear SAX1 elements available from the standard library, and each conical model has 500 elements along the defining meridian; trials using fewer three-noded quadratic elements makes little difference to the computational efficiency. The material has a typical Poisson's ratio of 0.3 and a Young's Modulus of $1 \mathrm{MPa}$, which is relatively soft but which intends to mimic a soft rubber, and all thicknesses are of the order of $0.1 \mathrm{~mm}$ for a cone side-length of around $50 \mathrm{~mm}$. The cone apex is replaced by a spherical cap of radius $3 \mathrm{~mm}$ which connects smoothly to the rest of the cone; a vertical force is applied normal to the cap at its pole, and the base of the cone is fully built-in. Geometrical nonlinearity is coupled with a "Riks" arc-length algorithm [7] during solution to capture fine, sometimes highly nonlinear features of the load path. Before examining a typical path, consider the four partially inverted cones in Fig. 2. They begin with a shallow cone in Fig. 2(a) inclined initially to the vertical by 
$60^{\circ}$ and become increasingly steeper with cone angles of $45^{\circ}, 30^{\circ}$, and $0^{\circ}$, which is a cylindrical tube. Each sub-figure also reveals the deformed cross-section where the crease profile is clearly evident and accentuated by highlighting elastic strain contours; each inverted cap is also highly strained. A sense of mirror symmetry about the ridge plane is also conveyed, in particular for the tube, where the inverted part is passing through its undeformed self. We have also added the Pogorelov outline to each sub-figure, in order to highlight the crease shape and its relative size as the cone angle decreases.

Representative results from the first case are given in Fig. 3. First, the inverting force, $F$, is plotted against the displacement of the apex, $d$, in Fig. 3(a). Although difficult to see, the response is linear at first before softening as the apical cap inverts fully, with the crease forming completely soon after. The crease then rolls down the side of the cone as the applied force decreases exponentially. When the crease is halted by the built-in base, the cone is almost inverted; at this stage, the crease mainly stretches in plane to accommodate $d$ increasing, leading to a rapid rise again in $F$ albeit with a small intermittent dip.

At some intermediate displacement, we plot the hoop-wise strain, $\epsilon_{\mathrm{h}}$, in Fig. $3(\mathrm{~b})$ as a function of the intrinsic coordinate, $s$, from the apex (Fig. 4(c)) which is the same for every meridian. Moving out of the inverted cap, there are high but localised strains which diminish quickly before increasing again mid-meridian at the position of the crease. There is first compressive hoop strain on the inside of the crease facing the inverted apex, followed by tensile strain on the outside. Such compression is surmised as a mechanism for secondary buckling of the crease but we note a strongly antisymmetrical profile about a local origin which exactly coincides with the ridge-line. The width of the crease can be clearly correlated with the span of major strain activity, and we choose bounding points to be given by $\epsilon_{\mathrm{h}}$ first becoming zero again on either side of the ridge-line. The corresponding meridional span is denoted as $2 l^{*}$, which will be compared to the same definition of width from theory. Notice also that the highest strain is around $0.5 \%$, which exceeds the yield strain of most metals; it is even higher at the start of inversion but decreases as inversion proceeds. Longitudinal strains are smaller but always vary in proportion to hoop strains by the Poisson's ratio, which suggests negligible meridional stresses compared to hoop stresses.

For each solution increment, we detect positions of zero strain in order to find the variation of $l^{*}$ with $d$, which is plotted in Fig. 3(c). We omit the data at small displacements because the crease has not yet localised, giving a false impression of its size. After becoming well formed, $l^{*}$ behaves in a regular way, increasing with $d$ at a diminishing rate. Such variation is repeated for many conical shapes and thickness, where the radius-to-thickness ratio of shell can range from 15 at the apex to nearly 1300 close to the base. We aim to predict the responsible factors 
for $l^{*}$ and so must carefully focus on the kinematic features in our model. But we also wish to find the force required to pull the crease through the cone. For example, it is not clear why the inverting force should decrease: at the same time, the crease circumference increases, suggesting that more of the cone is becoming strained viz. more external work and a higher force. We resolve this surmised discrepancy in the following analysis.

\section{Theoretical model}

Figure 4(a) shows a cross-section of the equivalent Pogorelov viewpoint of conical inversion. The inverted part can be reflected in the ridge plane to yield the original undeformed cone, and all lines are straight. This is the zero strain configuration because there are no changes in length anywhere, and zero shell thickness precludes any stored elastic energy in the ridge-line. More geometrical detail within the meridional plane is furnished in Fig. 4(b) for a cone with a sharp apex as well as our apical cap. This cone is also perfectly inverted under a polar displacement, $d$, with the crease - still of zero width - being located at a distance, $b$, along the initial meridian from the end of the cap. Using Figs 4(b) and (c), where the cone angle is denoted by $\alpha$ and the cap radius by $R$, simple geometry affords

$$
d=2 R(1-\sin \alpha)+2 b \cos \alpha
$$

The current latitudinal radius at the crease position is $r$, which is equal to $R \cos \alpha+b \sin \alpha$. At the same position, the current radius of hoop-wise curvature of the conical shell is measured by the distance from the vertical conical axis normal to the meridian, where the usual notation is $r_{2}$ from [8]. If we select the meridian in the upright part, then $r_{2}$ is drawn as per Fig. 4.2(b) and $r / r_{2}=\cos \alpha$. An explicit expression for $r_{2}$ in terms of $d$ can now be found by eliminating $b$ between them:

$$
r_{2}=R+\frac{\tan \alpha}{\cos \alpha}\left[\frac{d}{2}-R(1-\sin \alpha)\right]
$$

This returns $r_{2}=d \tan \alpha / 2 \cos \alpha$ for a perfect cone when $R$ is zero. Strictly speaking, the radius of curvature of the actual ridge-line is not defined because of the discontinuity in gradient there, and the expression for $r_{2}$ is only true immediately on either side of the ridge (if we had selected the inverted meridian instead, then $r_{2}$ changes direction and lies above $r$ ). But this presents no problem because at the ridge-line itself, we need to interrogate the local crease shape in order to ascribe curvature properties in detail.

Figure 4(c) shows a schematic axisymmetrical cross-section of a crease, which is drawn relative to the Pogorelov outline. Displacements away from this outline constitute straining and 
curving, where the normal component, $w$, is assumed to be dominant. We also assume that $w$ is symmetrical about the ridge-line because the crease is far enough away from the base or apex. The formulation can now be contracted slightly by defining a positive meridional coordinate, $x$, moving away from the ridge towards the base; $x$ does not take negative values, rather, any functions of it are merely reflected about $x=0$ either symmetrically or antisymmetrically provided we are careful about boundary conditions at $x=0$. The hoop-wise strain, $\epsilon_{\mathrm{h}}$, is therefore $w / r_{2}(x)$ from [8] where $r_{2}(x)$ is the variation in the underlying conical radius of curvature beyond the ridge-line, found by substituting $b$ with $b+x$ into Eqn 1 and so forth. The reason for the crease touching the Pogorelov ridge-line at $x=0$ is now apparent, for here $w$ must be zero, which sets the hoop-strain to be zero as per Fig. 3(b). Beyond the ridge-line, $r_{2}(x)$ increases, but if $w$ decreases sharply with $x$ then there is little change in $r_{2}$ within the crease itself: in other words, the radius of hoop-wise curvature of the crease is everywhere $1 / r_{2}$ with $r_{2}$ expressed by the current value of Eqn 2. As noted, meridional strains are not negligible, but $w$ does not depend on them directly. The curvature, however, in this direction, $\kappa$, is set by the shallow gradient expression, $\kappa=-\mathrm{d}^{2} w / \mathrm{d} x^{2}$, where absolute differentials assert the one-dimensional nature of problem.

We idealise further by excising half of the crease in Fig. 4(c) for all of $x$ and re-drawing it in Fig. 4(d). At the cut, $x=0$, and thus everywhere on the ridge-line, there must be a bending moment, $M$, and locally aligned forces in shear, $Q$, and in tension, $T$; these are all axisymmetrical quantities defined per unit length of shell and are drawn in their positive directions. Even though we do not show the far field forces at the base in this view, it is possible to write certain equilibrium relationships between them and $M, Q$ and $T$. However, specified in this way, our problem turns out to be statically indeterminate, and we must invoke arguments of geometrical compatibility for a complete solution. Very quickly, the level of algebraic complexity increases, which is not the aim of study. Instead, we assume that $T$ is not directly responsible for transverse displacements, so we neglect its effect for now, and that $M$ and $Q$ are wholly responsible for ensuring the local boundary conditions at $x=0$. We may therefore focus on the interaction of $M$ and $Q$ alone and, because $r_{2}$ is deemed to be constant everywhere along the crease, they behave as if they are loads applied to the edge of a cylinder of uniform radius, $r_{2}$.

In the absence of a normal pressure, the governing equation of transverse deflections for a cylindrical shell is given by [8]:

$$
\frac{\mathrm{d}^{4} w}{\mathrm{~d} x^{4}}+\frac{E t}{D r_{2}^{2}} w=0
$$

This is derived by considering equilibrium of an axisymmetrical element of original hoop-wise curvature, $1 / r_{2}$. Linear elasticity is specified and the deformation is controlled by meridional 
bending where $M=D \kappa$, and by hoop-wise straining via $\epsilon_{\mathrm{h}}: D$ is the standard flexural rigidity equal to $E t^{3} / 12\left(1-\nu^{2}\right)$ where $E$ is the Young's modulus, $\nu$ is the Poisson ratio, and $t$ is the transverse shell thickness. A slightly more compact notation replaces the $w$ pre-factor with $\beta^{4}=E t / 4 D r_{2}^{2}$, and we try solutions of the form, $w=A e^{\xi x}$, where the unknown amplitude, $A$, is found later from the boundary conditions. The corresponding auxiliary equation sets $\xi^{4}+4 \beta^{4}=0$, returning roots of $\xi=( \pm 1 \pm \sqrt{-1}) \beta$ and a general solution of four terms as $A_{1} e^{\beta x} \sin \beta x+A_{2} e^{-\beta x} \sin \beta x \ldots$ etc. Those terms involving exponential growth can be discounted for $w$ rapidly decaying with $x$, resulting in two terms whose amplitudes are found by setting $M=D \kappa$ and $Q=\mathrm{d} M / \mathrm{d} x$ at $x=0:$

$$
w=\frac{e^{-\beta x}}{2 \beta^{2} D}\left[M(\sin \beta x-\cos \beta x)+\frac{Q}{\beta} \cos \beta x\right] \text { for } x \geq 0
$$

Ensuring that $w=0$ at $x=0$ obviates $M=Q / \beta$, giving a gradient of $M / 2 \beta D$ at the same position which must equal $\pi / 2-\alpha$ from Fig. $4(\mathrm{~d})$. The final expression for $w$ turns out to be rather compact:

$$
w=\frac{(\pi / 2-\alpha)}{\beta} e^{-\beta x} \sin \beta x \text { for } x \geq 0
$$

Because of the proportionality between $w$ and hoop strain $\epsilon_{\mathrm{h}}$, and given the latter's variation in Fig. 3(b) on either side of the ridge-line, we could have inferred the expression above by inspection. The formal analysis is conclusive and Eqn 5 immediately offers $l^{*}$ when we set $\epsilon_{\mathrm{h}}$, hence $w$, equal to zero at $x=l^{*}$, giving $\sin \beta l^{*}=0$ and $\beta l^{*}=\pi$. Recalling our previous definitions of $\beta$ and $D$, we can therefore write:

$$
l^{*}=\pi\left[\frac{4 D r_{2}^{2}}{E t}\right]^{1 / 4} \Rightarrow l^{*}=\frac{\pi r_{2}^{1 / 2} t^{1 / 2}}{\left[3\left(1-\nu^{2}\right)\right]^{1 / 4}} \quad\left(\text { and } l^{*} \approx 2.44 \sqrt{r_{2} t} \text { for } \nu=0.3\right)
$$

This confirms that the crease increases in width during inversion because $r_{2}$ increases with $d$ according to Eqn 2. Comfortingly, the square-root dependency of $l^{*}$ upon $r_{2}$ points to the cusplike variation we expect, in particular, when $R$ is small enough to be ignored, $r_{2}$ is proportional to $d$ for a given cone angle and thus, $l^{*}$ varies with $\sqrt{d}$. In general, being proportional to the square root of the product of thickness and local radius of curvature, $l^{*}$ is akin to the width of boundary layer deformation along the edges of long, curved thin-walled strips [9] and to the width of periodic compression "wrinkles" in a variety of pre-tensioned membranes [10, 11], as discussed later.

We compare Eqn 6 to finite element data in Fig. 5 first for a fairly shallow cone $\left(\alpha=60^{\circ}\right)$ of three different thicknesses, $t=0.2 \mathrm{~mm}, 0.1 \mathrm{~mm}$ and $0.067 \mathrm{~mm}$. For the apical cap, $R=3 \mathrm{~mm}$, and the straight meridian is $50 \mathrm{~mm}$ long. We plot dimensionless quantities $l^{*} / t$ vs $d / d_{\max }$ where 
$d$ is Eqn 1 and $d=d_{\max }$ when the cone is fully inverted. Again, early computational data near $d=0$ is omitted because the crease has not yet properly formed. Otherwise, the predictions by Eqn 6 are almost indistinguishable from those of finite elements. We also plot the variation of $l^{*} / r_{2}$ against $d / d_{\max }$ to determine how the crease width compares to the local radius of conical curvature. The ratio $l^{*} / r_{2}$ is always a maximum initially when $r_{2}$ is smallest, and is typically around $0.25-0.4$, giving a crease length, $2 l^{*}$, just slightly less than $r_{2}$. But the ratio quickly attenuates to around $0.1-0.15$, which implies that $r_{2}$ changes little over the crease span, thereby confirming our original proposal.

Figure 6 deals with different cone angles of the same thickness and, again, the differences between theory and computational data are minimal. Even though a steeper cone implies a slower rate of change of $r_{2}$ along a meridian, which bolsters the cylindrical shell analogy, the initial gradient in Fig. 4(d) at $x=0$ also increases, which undermines the shallow shell assumption. For a tube where $\alpha=0^{\circ}$, this gradient is infinite yet Eqn 6 differs by less than $10 \%$ compared to finite element data, which is remarkable. In this case, $r_{2}$ is a constant and equal to the radius of the original apical cap. Note that in general, as the cone angle decreases, so does $l^{*}$.

We now proceed to finding the inverting force, $F$, using an energy formulation for the entire cone based on the strain energy stored in the crease and not in the deformed apical cap. This process is reasonably light on algebra because $w$ is a fairly simple expression and there are only two major strain energy components; as we remarked before on statical indeterminacy, finding $F$ by equilibrium etc. becomes unwieldy very quickly. First, let the amplitude of $w$ in Eqn 5 be $A$ for convenience, which gives:

$$
\epsilon_{\mathrm{h}}=\frac{w}{r_{2}}=\frac{A}{r_{2}} e^{-\beta x} \sin \beta x, \quad \kappa=-\frac{\mathrm{d}^{2} w}{\mathrm{~d} x^{2}}=2 A \beta^{2} e^{-\beta x} \cos \beta x
$$

An expression for the strain energy density per unit surface area of cone may now be distilled from the general expression for shells found in [8] by assuming that these kinematic terms are predominant and remembering that in-plane meridional strains are equal to $-\nu \epsilon_{\mathrm{h}}$. Hoop-wise curvature changes are negligible compared to $\kappa$, and, because of axisymmetry, shear stresses and twisting curvatures are zero. The simplified density can be verified as $(1 / 2) E t \epsilon_{\mathrm{h}}^{2}+(1 / 2) D \kappa^{2}$, and the total strain energy is then found by integrating this expression over the conical surface area where, recall, $x$ is only positive. The integration can be simply doubled because the crease shape is perfectly symmetrical about the ridge-line. Its limits are clearly zero and $x=l^{*}$ but we if set the upper limit to infinity instead, the resulting expressions are greatly simplified but remain accurate because of the rapid attenuation of $w$. To this end, we calculate the identities: 


$$
\int_{x=0}^{x=\infty}\left(e^{-\beta x} \sin \beta x\right)^{2}=\frac{1}{8 \beta}, \quad \int_{x=0}^{x=\infty}\left(e^{-\beta x} \cos \beta x\right)^{2}=\frac{3}{8 \beta}
$$

and note that a hoop-wise elemental strip has an area of $2 \pi r_{2} \cos \alpha \mathrm{d} x$ where, as per usual, we set $r_{2}$ equal to its value at the ridge-line. Bringing altogether, we find the total strain energy stored in the cone, $U$, to be

$$
U=\frac{2 \pi E t A^{2} \cos \alpha}{8 r_{2} \beta}+3 \pi r_{2} D A^{2} \beta^{3} \cos \alpha \quad \text { with } A=(\pi / 2-\alpha) / \beta
$$

The total potential energy for the system, $V$, is defined as the difference between the internal strain energy and quantities of external work. Since the point of application of $F$ moves by $d$ in the same direction, the latter is simply $F d$, and $V=U-F d$. We may retain $d$ in its general form but for compactness we set $R$ to be zero, giving $d=2 r_{2} \cos \alpha / \tan \alpha$. The expression for $V$ is now

$$
V=\frac{\pi \cos \alpha(\pi / 2-\alpha)^{2} E t^{5 / 2} r_{2}^{1 / 2}}{3^{3 / 4}\left(1-\nu^{2}\right)^{3 / 4}}-\frac{2 F r_{2} \cos \alpha}{\tan \alpha}
$$

after substituting for $A$ and replacing $\beta$ with $\pi / l^{*}$. $F$ is now found by minimising $V$ with respect to the generalised coordinate, $r_{2}$, and setting equal to zero. After some manipulation and reexpressing $r_{2}$ in terms of $d$, we can write the final expression for $F$ in one dimensionless form as:

$$
\frac{F}{E t^{2}}=\frac{\pi(\sin \alpha)^{1 / 2}(\pi / 2-\alpha)^{2}}{2^{3 / 2} 3^{3 / 4}\left(1-\nu^{2}\right)^{3 / 4}} \cdot \frac{t^{1 / 2}}{d^{1 / 2}}
$$

We compare this to finite element data in Fig. 7 for cones of increasing steepness up to $\alpha=15^{\circ}$. We cannot compare meaningfully to the case of a tube $(\alpha=0)$ because Eqn 11 returns zero force owing to the $\sin \alpha$ term when the finite element analysis gives a non-zero but constant force. The reason for the latter stems from having an apical cap, where a force must be applied to keep it inverted even though there is no energy penalty as the crease moves along the tubewhich is reflected by Eqn 11. For the cone angles in Fig. 7, we include the computational data during the formation stage, in order to highlight the much higher forces at this time. When the crease becomes well formed afterwards, the correlation is delightfully close despite the data beginning to diverge for the steepest cone. We plot the dimensionless displacement as $d / t$ to compare directly with Eqn 11 although it can easily be $d / d_{\max }$, as in the other figures. Because of the reciprocal term, $d^{-1 / 2}, F$ must decay even though we initially thought it may increase as the crease envelopes more material during displacement. Note that Fig. 7 deals with the same thickness, $0.1 \mathrm{~mm}$ : analysis for more thicknesses show the same close trends but are not included for brevity's sake. We re-plot the data from Fig. 7 in Fig. 8 using logarithmic axes to ascertain 
the power-law variation of the computational data assuming that such a variation is presently applicable. We process only the data beyond formation of the crease, and we then add the best fit predictions to the figure; for each case, the power-law index, tantamount to the gradient of the data, is given. There are two comforting findings. First, the force $v s$ displacement data is "linear" in the logarithmic sense, thereby confirming the sensibility of a power-law variation. Second, all indices are very close to -0.5 as suggested by Eqn 11, which reinforces the credibility of this formula and underlying assumptions.

\section{Discussion}

Despite employing a linear governing equation of deformation for shallow shells, our analysis accurately captures the steady state crease size and inverting force over a range of initial conical geometries. It is especially pertinent for small conical angles where, despite the initial geometry being close to cylindrical, the boundary condition for rotation is evidently not small. We note first that $l^{*}$ does not depend on this rotation. Secondly, the form of the shallow transverse deflections, $w$, in Eqn 5 turns out to be a rather good "fit" for the large rotation nature of this specific problem for several reasons. From the arc-tangent of the gradient at $x=0$ we may compare the corresponding boundary rotation against the actual value, $\pi / 2-\alpha$. For a pure cylinder $\left(\alpha=0^{\circ}\right), \mathrm{d} w / \mathrm{d} x=\pi / 2$ giving a rotation of $\arctan (\pi / 2) \approx 58^{\circ}$; however, as the cone angle increases, the discrepancy between these angular measures quickly reduces e.g. when $\alpha=45^{\circ}$, we see a rotation of $\arctan (\pi / 2-\pi / 4)=38^{\circ}$. Moderate rotations are therefore described very well. Away from the boundary, the proportions of crease afforded by $w$ also compare well to finite elements: for example, the ratio of $w_{\max }$ to $l^{*}$ is largest for a cylindrical tube and is roughly equal to one-fifth from finite element data; Eqn 5 predicts $0.5 \exp (-\pi / 4) \sin \pi / 4 \approx 1 / 6$. The inverting force, Eqn 11 stems from a Rayleigh-Ritz type energy analysis, which is known to be accurate if the selected mode-shape is close to actual deflections, especially so when $w$ comprises a single term. Of course, we could approach the problem within the spirit of large deflections from the outset. The corresponding governing equation is a cylindrical version of the well-known beam elastica, where geometrical nonlinearity - particularly in view of axial force effects - is central to its expression. However, for our problem, the effect of axial forces is not dominant because their stresses are much smaller than circumferential stresses; this can be seen in the shape of the crease about the ridge line, which is virtually symmetrical because in-plane meridional stresses are minimal (but not zero). As noted initially, many scaling analysis do not consider the detailed variation of crease shape, which does not reveal the pre-factor for any characteristic length, even though its dimensional dependencies are clear: suffice to say, we have 
established the pre-factor immediately.

Our linear analysis is also helped by the crease being a relatively small but concentrative feature within the overall geometry, which allows us to simplify its local performance; it is also a contrived example which permits controlled formation of a uniform crease. Apart from the case of spherical inversion with its own circular crease, similar problems in the literature usually focus on creases formed in an unregulated way e.g. when a paper sheet is manually crumpled into a ball. If the ball is unravelled into a planar sheet again, the crease distribution is naturally random but all creases are straight [12]. As such, we may be tempted to think these are physically different creases compared to ours; however, their analysis often embraces the same competition between local bending and stretching where the simplest stress-free crease begins as a narrow cylindrical strip before being bent into a shallow saddle along its axis in order to mimic the effect of global bending [13]. The strip becomes doubly curved, which incurs stretching according to Gauss' Theorema Egregium [8], and the inevitable balance between these deformation modes results in a natural radius of span-wise curvature for the crease. Our creases are also doubly curved because they are toroidal and, using the Pogorelov viewpoint, we see how they are stretched: and if we return to the strain energy component in Eqn 10 and re-insert the flexural rigidity, $D$, into this expression, we find that the energy stored in our creases varies as $D\left(r_{2} / t\right)^{1 / 2}$. For the straight creases in [13], this variation has been shown to go as $D(L / t)^{1 / 3}$, where $L$ is the axial length of the crease. In essence, $r_{2}$ also measures the length of our creases, so we see a common ratio of length-to-thickness but with quite different power law exponents. This comparison could be serendipitous but it begs an obvious and interesting research question for future study. One approach might be to combine what is known about the bending of straight creases, with that of forming conical creases, in a generalised way. Finally, we note that it is not surprising that our single conical crease is geometrically similar to the repetitive, periodic wrinkles in buckled thin-walled plates or membranes. Indeed, in [10], they remark from an energy perspective that a characteristic wavelength requires "ingredients" equivalent to the bending of a plate on an elastic foundation: such problems are well known to be governed by a fourth-order linear differential equation of similar form to Eqn 3 [8]. Thus, we would expect the same nature and dimensional variation in our solution.

\section{Acknowledgements}

The author first worked on this problem in September 2012 during a visiting research tenure at the University of Pierre and Marie Curie (UPMC) in Paris. He is extremely grateful to Prof. Corrado Maurini for making this stay and its associated "head-space" happen. Finally, the 
author is grateful to one of the anonymous referees for emphasising the comparison to problems involving periodic wrinkling of thin plates and membranes.

\section{References}

[1] A V Pogorelov. Bending of Surfaces and Stability of Shells. Translations of Mathematical Monographs: American Mathematical Society, 1988

[2] E Zhu, P Mandal and C R Calladine. Buckling of thin cylindrical shells: an attempt to resolve a paradox. International Journal of Mechanical Sciences 44(8): 1583-1601, 2002.

[3] M Gomez, D E Moulton and D Vella. The shallow shell approach to Pogorelov's problem and the breakdown of 'mirror buckling'. Proceedings of the Royal Society A, 453: paper $20150732,2016$.

[4] S Knoche and J Kierfeld. Secondary polygonal instability of buckled spherical shells. EPL (Europhysics Letters), 106(2): paper 24004, 2014.

[5] P K Gupta. A study on inversion of metallic thin-walled conical shells. International Journal of Crashworthiness, 16(6): 607-619, 2011.

[6] A Ramachandran. First Year PhD Report, Department of Materials Science, University of Cambridge, 2013.

[7] ABAQUS Users Manual Version 6.11. Dassault Systemes, Providence, RI, USA, 2011.

[8] C R Calladine. Theory of Shell Structures. Cambridge University Press, 1983.

[9] E H Mansfield. The Bending and Stretching of Plates. Cambridge University Press, 1989.

[10] E Cerda and L Mahadevan. Geometry and physics of wrinkling. Physical Review Letters, 90(7): paper 074302, 2003.

[11] Y W Wong and S Pellegrino. Wrinkled membranes part II: analytical models. Journal of Mechanics of Materials and Structures, 1(1): 25-59, 2006.

[12] M Ben Amar and Y Pomeau. Crumpled paper. Proceedings of the Royal Society A, 453(1959): 729-755, 1997.

[13] A E Lobkovsky and T A Witten. Properties of ridges in elastic membranes. Physical Review E, 55(2): 1577-1589, 1997. 


\section{$5 \quad$ Figures}

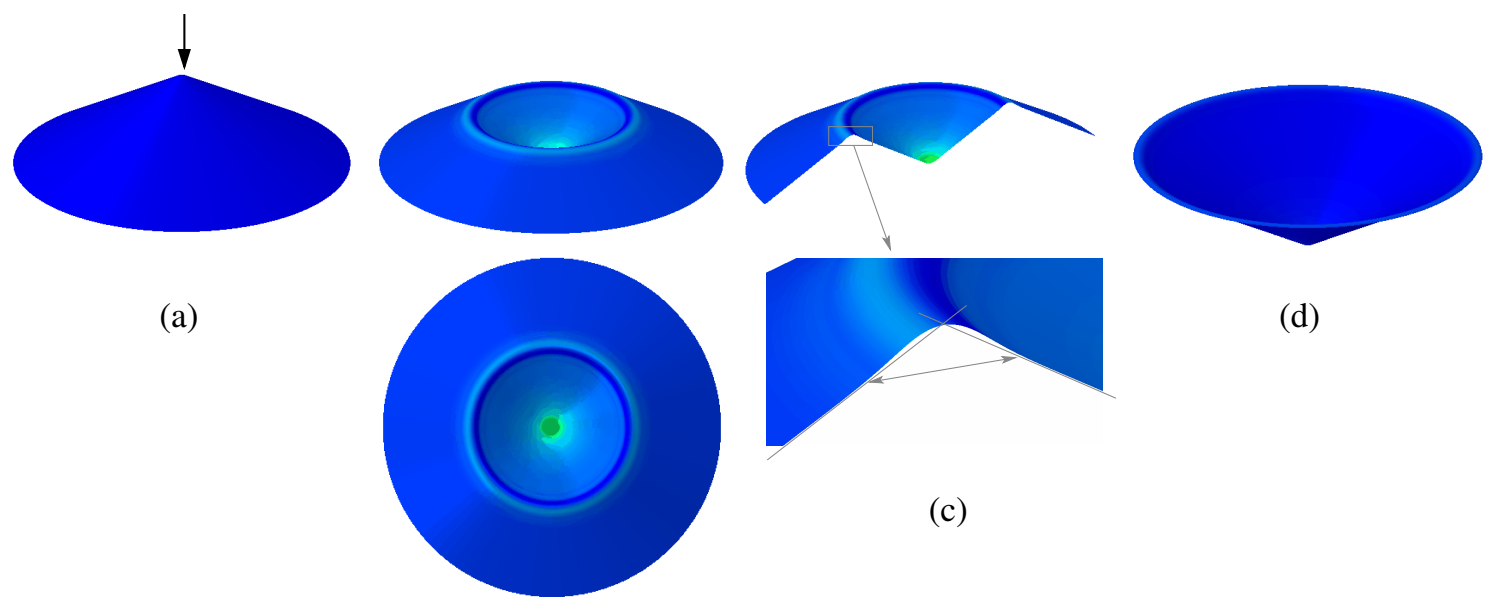

(b)

Figure 1: Elastic inversion of a thin conical shell using finite element analysis. The initial cone angle, measured from the vertical, is $60^{\circ}$, and the side-length of cone is $50 \mathrm{~mm}$; the apex is a small spherical cap of radius $3 \mathrm{~mm}$, and everywhere the thickness is $0.1 \mathrm{~mm}$. (a) Initial configuration where a force is applied to the apex and the base of the cone is held rigid. (b) Two views of the partially inverted state, highlighting contours of maximum principal strain. These are focussed in the original apex and around the ridge which separates the inverted part from the original upright part. (c) Cutaway view of (b) from afar and close-up, showing the deviation of the conical shape near the ridge-line away from perfectly straight meridians (grey lines). The width of this deviation is roughly the span over which the strain contours diminish in variation, and shown by the arrow: this is the conical "crease" associated with the ridge-line. (d) Almost fully inverted cone, indicating the extent of deformation during analysis. 


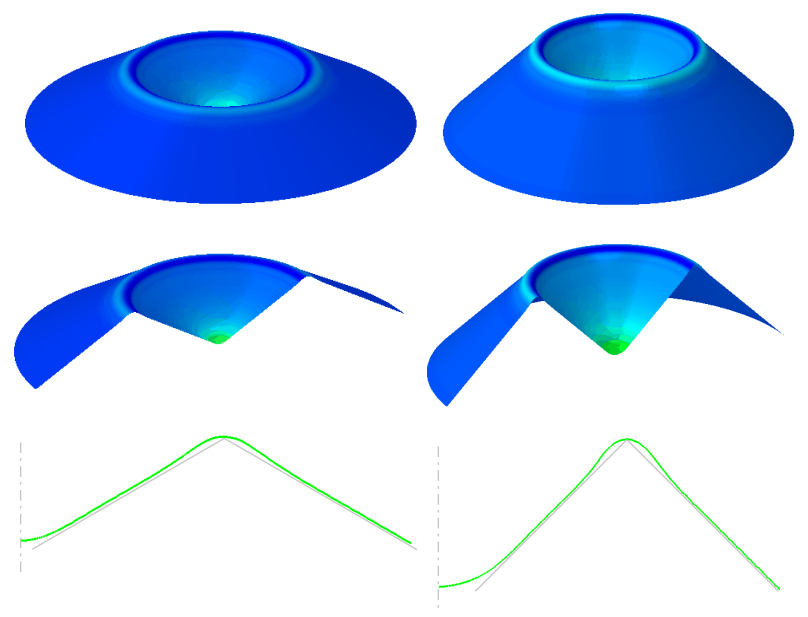

(a)
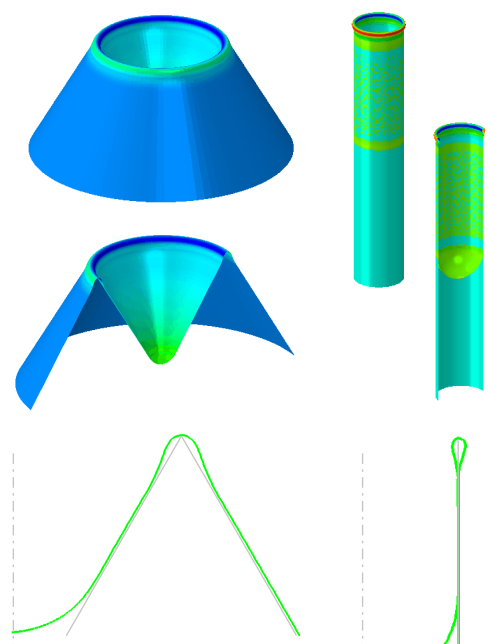

(c)

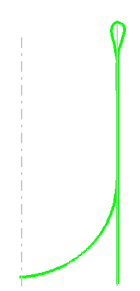

(d)

Figure 2: Partial inversion of four different cones using finite elements. Top row: full cones partially inverted. Middle row: cutaway counterparts highlighting the crease width around the ridge-line according to colour changes in the maximum principal strain contours. Bottom row: axisymmetrical meridians (green) compared to the perfectly straight meridians (grey), showing how the actual crease region diverges and "detaches" from the latter; the crease width is later defined to be the arc-length of the detached region. The cone angles are (a) $60^{\circ}$, (b) $45^{\circ}$, (c) $30^{\circ}$ and (d) $0^{\circ}$; other material and geometric properties are the same as Fig. 1. In (d), the inverted part overlays the original undeformed tube, giving a mottled complexion of interference. 

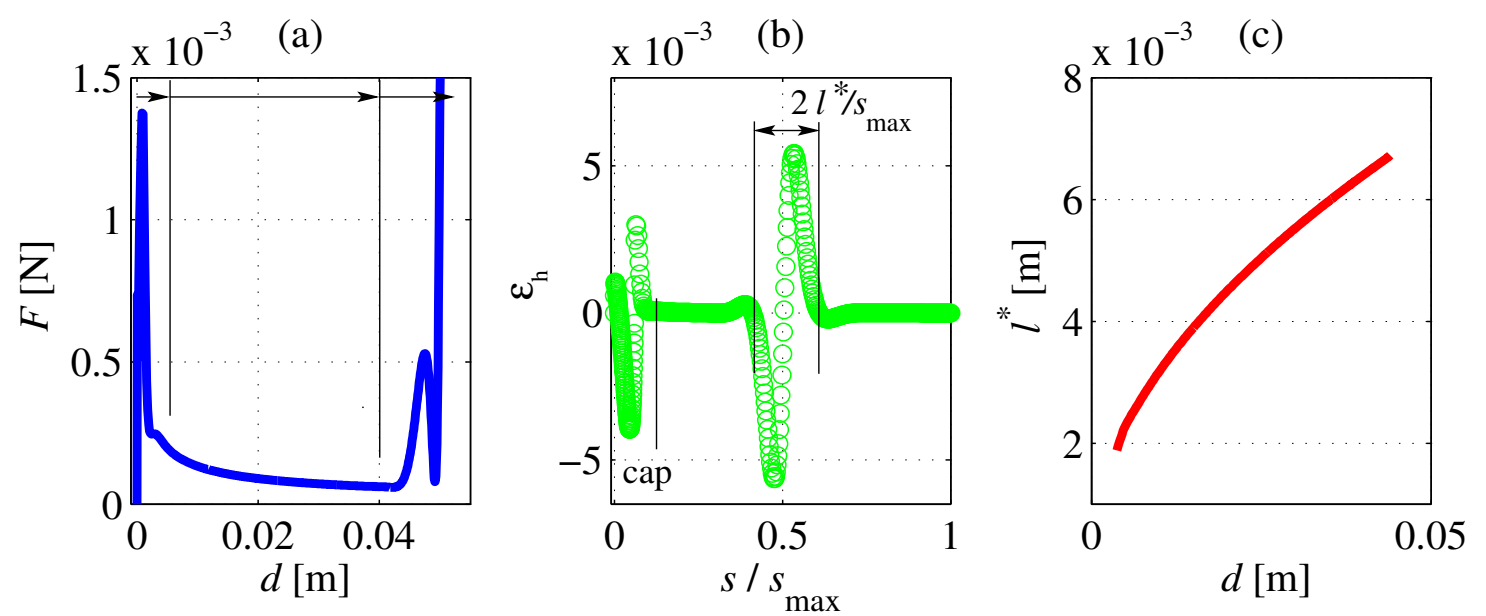

Figure 3: Properties of the conical inversion of Fig. 1. (a) Inverting force, F, vs apex displacement, $d$. Three regimes are highlighted: first, initial inversion up to $d \approx 0.006 \mathrm{~m}$ where the apical cap turns inside out and the crease on the ridge-line becomes fully formed: second, movement of the crease through the undeformed cone as inversion proceeds up to $d \approx 0.04 \mathrm{~m}$; third, cessation of inversion when the crease reaches the base of the original cone. (b) Hoop-wise strain, $\epsilon_{\mathrm{h}}$, along a meridian at some point during inversion when the crease is well formed. The meridional coordinate from the apex is $s$, see Fig. $4(\mathrm{c})$, and $s_{\max }$ corresponds to the base of the cone. Up to $s / s_{\max } \approx 0.1$, the strains belong to the original apical cap; around $s / s_{\max }=0.5$, we encounter strains in the crease, which diminish quickly on either side. This pronounced variation gives a strong indication of the extent of the crease: it is antisymmetrical about the ridge-line with $\epsilon_{\mathrm{h}}=0$ on the ridge-line itself; after peaking and reaching zero on either side, $\epsilon_{\mathrm{h}}$ quickly diminishes. We define the width of the crease by the span, $2 l^{*}$, between these zero-points around the ridge-line. Green circles are distinct nodal positions along the meridian from finite element analysis, which are used to calculate this width at each increment of solution. (c) Variation of $l^{*}$ with $d$ during inversion. The initial data before $d \approx 0.005 \mathrm{~m}$ is not shown because the crease has not yet separated from the apical cap. Note the diminishing rate by which $l^{*}$ increases. 


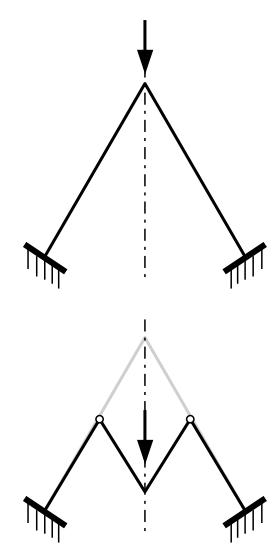

(a)

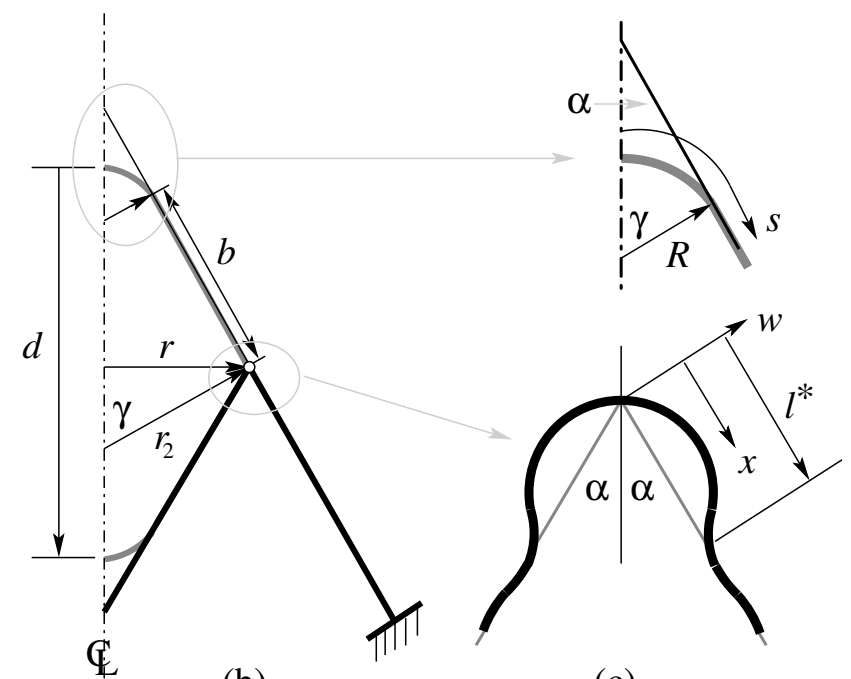

(b) (c)

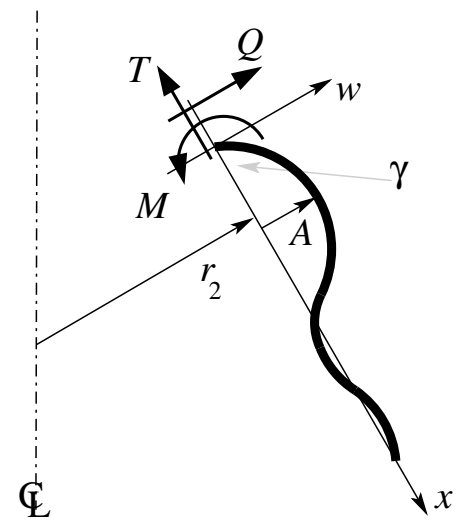

(d)

Figure 4: Schematic rendering of a partially inverted cone and its ridge-line crease. (a) The "Pogorelov" viewpoint of deformation in which the cone inverts symmetrically under an axial force. The small circles signify the position of the inverting ridge-line. (b) Meridional geometry for perfect inversion. The heavy black line is a cone with a sharp apex; the grey line is our cone with a shallow apical cap whose vertical displacement is $d$. For both, the position of the ridgeline is a small circle located a distance $b$ from the edge of the cap and sitting at radius, $r$, from the centre-line (CL) axis of revolution. The radius of hoop-wise curvature at the ridge for a cone is $r_{2}$, which extends from the centre-line to the undeformed meridian immediately below the ridge; $\gamma$ is a dummy angle equal to $\pi / 2-\alpha$, where $\alpha$ is the cone angle. (c) Top: definition of cap geometry from (b) where $R$ is its radius of curvature; $s$ is an intrinsic coordinate from the top of the cap. Bottom: Close-up view of the crease geometry around the ridge-line. The underlying straight axes is the Pogorelov shape from (a), which intersects the crease ridge-line. The local coordinate $x$ is measured from the ridge-line on one-side only; $x=l^{*}$ is the characteristic halflength of crease when the transverse displacement, $w$, becomes zero again. (d) Half of the crease from (c) with free-body forces and bending moment added to the position of the cut at $x=0$. The positive directions of $M$ and $Q$ give $\mathrm{d} M / \mathrm{dx}=Q$. 
(a) $t=0.2 \mathrm{~mm}$
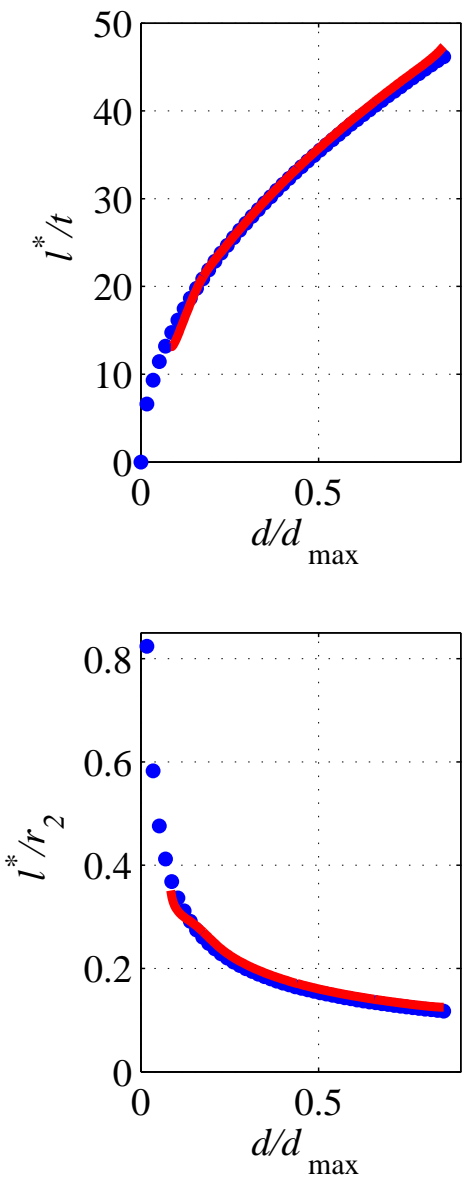

(b) $t=0.1 \mathrm{~mm}$
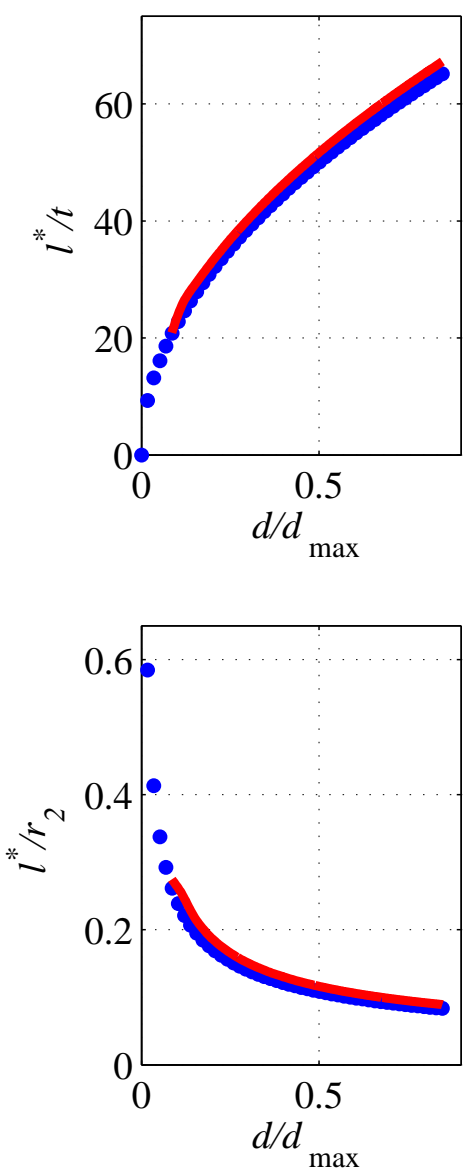

(c) $t=0.067 \mathrm{~mm}$
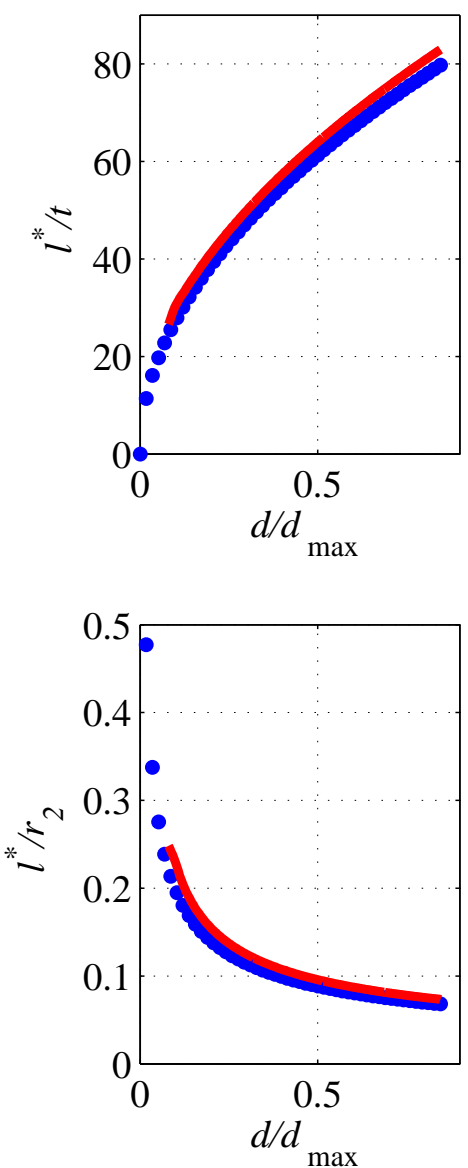

Figure 5: Comparison of $l^{*}$ from Eqn 6 (blue dots) with finite element data (solid red line). Each vertical pair of sub-figures is for the same indicated thickness, and all have the same cone angle, $\alpha=60^{\circ}$, and the same apical cap radius, $R=3 \mathrm{~mm}$; the Young's modulus is $1 \mathrm{MPa}$ and the Poisson's ratio is 0.3 . The top row compares the crease width, $l^{*}$, to shell thickness, $t$; the bottom row considers $l^{*}$ with the local radius of conical curvature, $r_{2}$. The apex displacement is $d$, which reaches a maximum value, $d_{\max }$, when the cone is fully inverted. The early parts of all finite element data do not produce distinct trends because the crease is not properly formed, so are omitted for clarity. 
(a) $\alpha=60^{\circ}$

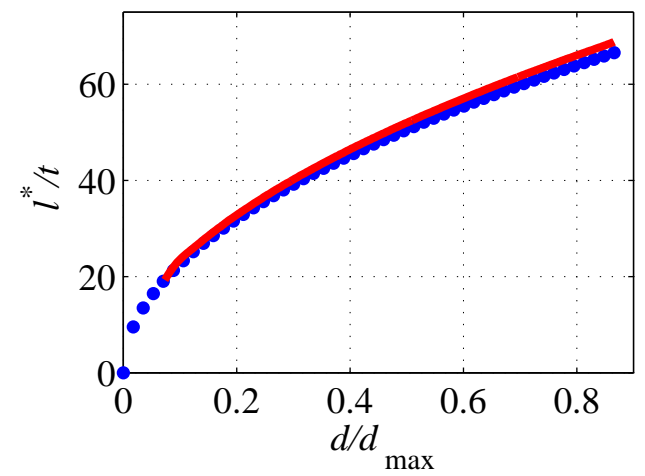

(c) $\alpha=30^{\circ}$

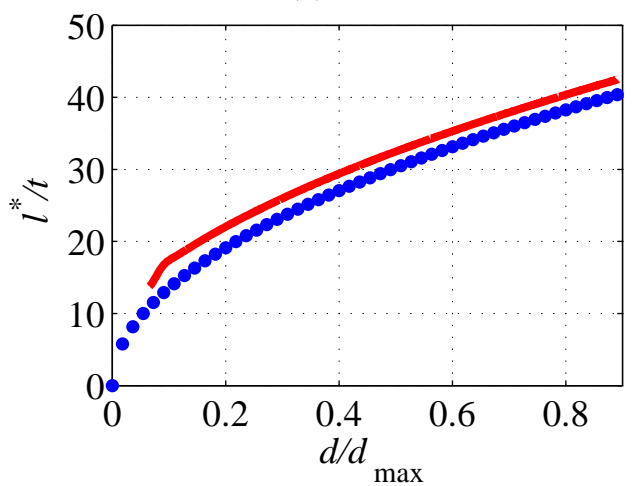

(b) $\alpha=45^{\circ}$

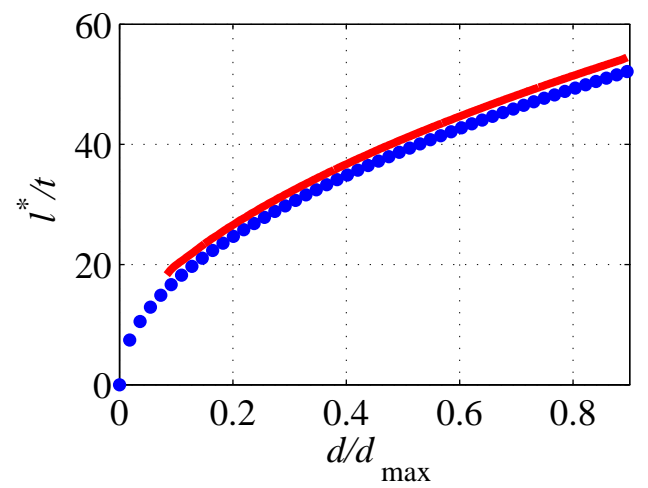

(d) $\alpha=0^{\circ}$

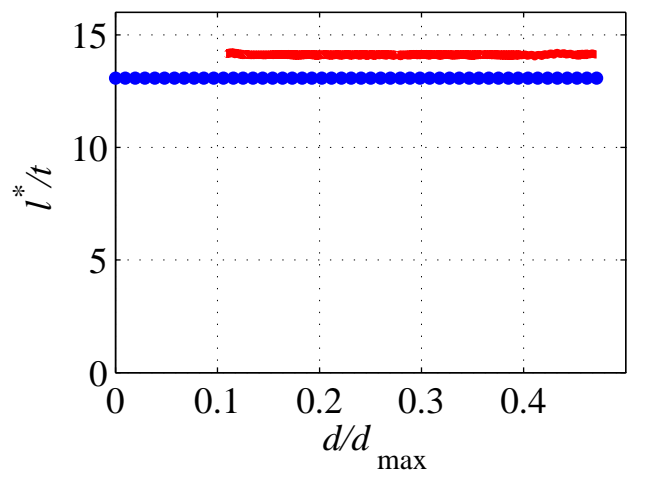

Figure 6: Comparison of $l^{*}$ from Eqn 6 (blue dots) with finite element data (solid red line) for different indicated cone angles. The shell thickness for all is $0.1 \mathrm{~mm}$, the apical cap has a radius of $3 \mathrm{~mm}$, and the material properties are the same as Fig. 5. Again, early finite element data does not apply and is left out, and because (d) is a tube, we do not show all of $l^{*}$ with respect to $d$ because it is practically constant. 
(a) $\alpha=60^{\circ}$

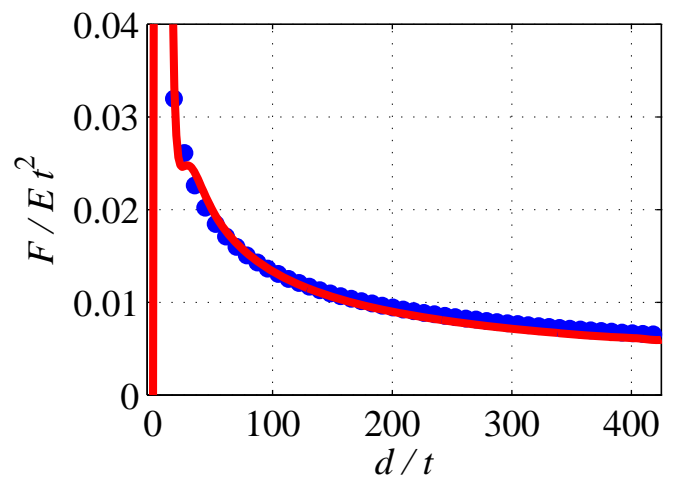

(c) $\alpha=30^{\circ}$

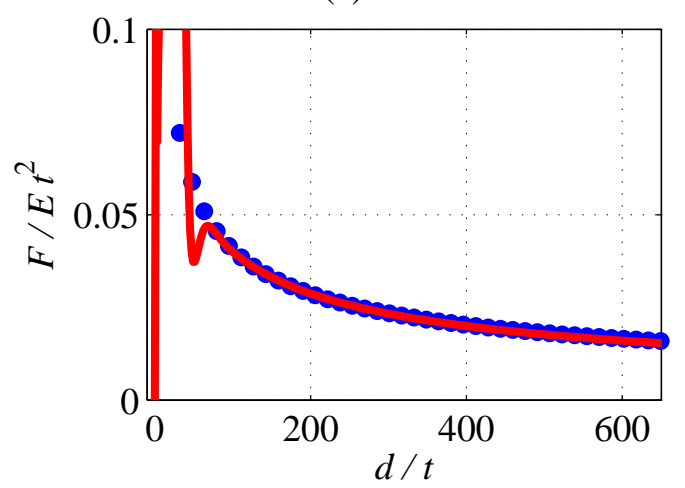

(b) $\alpha=45^{\circ}$

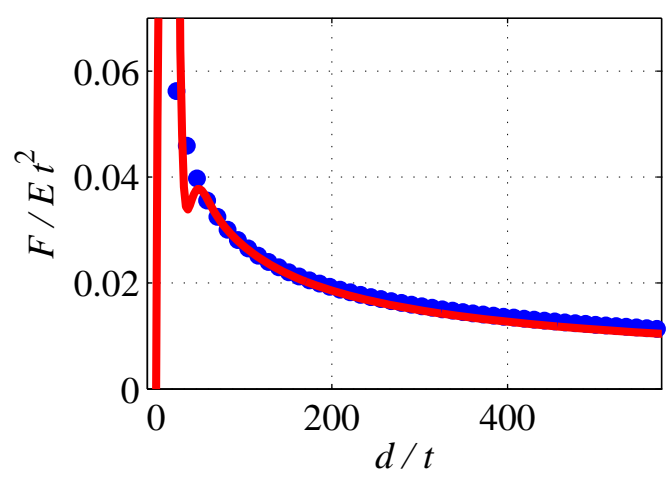

(d) $\alpha=15^{\circ}$

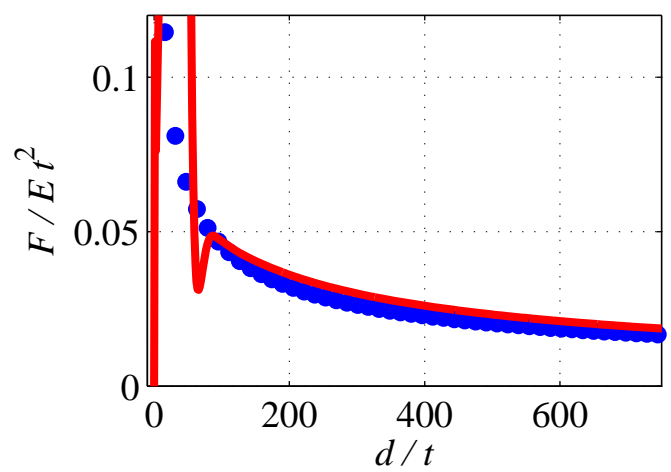

Figure 7: Comparison of the inverting force, $F$, from Eqn 11 (blue dots) with finite element data (solid red line) under displacement, $d$, for the indicated cone angles, $\alpha$. The Young's modulus, $E$, is $1 \mathrm{MPa}$ and the Poisson's ratio, $\nu$, is 0.3 ; all thicknesses are $t=0.1 \mathrm{~mm}$. 

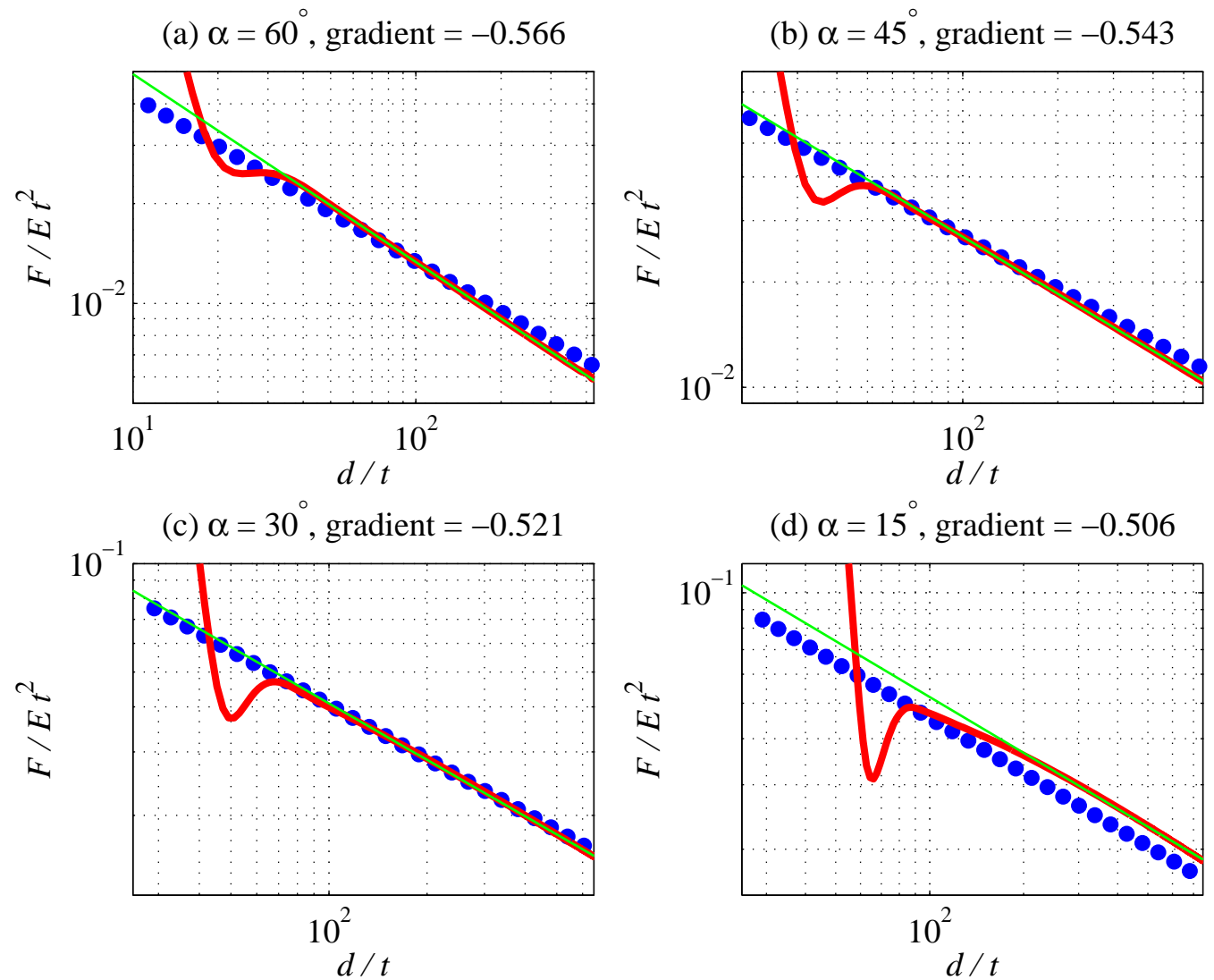

Figure 8: The same force-displacement data from Fig. 7 plotted on logarithmic axes: as before, blue dots are for theory and solid red lines for finite element data. Solid green lines pertain to the best power-law "fit" of the finite element data for a well-formed crease where it is assumed $F \propto d^{m}$; the corresponding index, $m$, which is also the gradient of green lines, is indicated in each sub-title. 